Nurse Practitioner Open Journal

DOI 10.28984/npoj.vi2.361

\title{
Body Image Disturbance in Female Adolescents Using Online Learning Platforms: A Concept Analysis
}

NPOJ 2022, pp. 15 - 44

Sauyoung Lee MN, NP-PHC ${ }^{1}$

sauyoung.lee@ryerson.ca

Erin Ziegler PhD, NP-PHC ${ }^{1}$

eziegler@ryerson.ca

1. Daphne Cockwell School of Nursing, Ryerson University, Toronto, Ontario, Canada

\begin{abstract}
Aim

To analyze the concept of body image disturbance in female adolescents using online learning platforms as a phenomenon of interest to nurse practitioners and other health care practitioners.

\section{Background}

With the declaration of the COVID-19 pandemic, the use of online learning platforms as a primary mode of learning has increased exponentially among adolescents. While research is still lacking in this field, the literature on traditional social media suggests that these online learning platforms may negatively influence body image and emotional outcomes. Particularly vulnerable to these outcomes are female adolescents, whose construction of own body image is highly influenced by their self-perceived evaluation from their peers. While the concept of body image disturbance is well defined in adolescent eating disorders, it has not been characterized in the context of online learning platforms. Defining the concept of body image disturbance in this context is crucial for recognizing its occurrence and providing early intervention.

Methods

The Walker and Avant method of concept analysis was used to analyze the concept.

Findings

The defining attributes of body image disturbance among female adolescents using online learning platforms are: (1) Feeling displeasure with the perceived appearance while using video streamed platforms; (2) Self-surveillance of one's appearance; and (3) Upward comparison of one's appearance with others and experiencing feelings of inadequacy.

Conclusion

As COVID-19 continues to disrupt the traditional school experience, nurse practitioners can use the presented scenarios, along with examples of questionnaires noted in this study, to recognize and delineate the occurrence of body image disturbance in female adolescents using online learning platforms. A development of a standardized definition of the concept will enable nurse practitioners to recognize its occurrence and to provide interventions in a timely manner.
\end{abstract}

\section{Keywords}

Adolescent, body image, social media, video chatting, nursing, nurse practitioner, psychological distress, online learning 


\section{Introduction}

The topic of body image disturbance has garnered increasing attention especially since the declaration of the coronavirus disease 2019 (COVID-19) pandemic and a resulting shift to a new form of social media - online learning platforms (World Health Organization, 2020; United Nations Educational, Scientific and Cultural Organization, 2020). In response to the pandemic, Ontario public schools transitioned to Board-selected learning management systems, including D2L Brightspace Learning Platform, Google Classroom, Zoom, and Microsoft Teams (Government of Ontario, 2020; 2021; Paglinawan, 2020). These platforms all have a common feature of live synchronous classes which include live streamed videos of oneself that are displayed alongside their classmates (D2L, 2020). Unlike in-person interactions, students are now able to overtly, and yet discreetly, evaluate their own and classmates' appearances in real time as they interact with each other. Owing to the limitations of technology, webcams, as with smartphones, distort facial and body proportion providing inaccurate representation of individuals' appearance (Cristel et al., 2020; Třebický et al., 2016). The transition to online learning platforms has inadvertently exposed students to prolonged durations of digitally distorted-reflections of themselves.

While there are few studies on the effects of online learning on body image, its potential influence on students can be drawn from research in related fields, such as traditional social media. Literature suggests that social media can lead to a negative body image and emotional outcomes including depressive symptoms (Paxton et al., 2006; Siegel, 2002), and low selfesteem (Fox et al., 2021; Mills et al., 2018; Vall-Roqué et al., 2021). Adolescents are especially vulnerable to these effects as they are at a critical stage of developing their body image (Hogan $\&$ Strasburger, 2008). How they are perceived by their peers becomes an important part of their evaluation of self and construction of their body image (Elkind, 1967). Female adolescents appear to be especially susceptible, regardless of their race or ethnicity (Siegel, 2002). By the early adolescent years, up to half of all females' report concerns of being overweight despite many having a healthy weight (Hogan \& Strasburger, 2008). Overall, video-based online learning platforms may have negative influences on female adolescents.

While body image disturbance may be a common issue among female adolescents using video-based online learning platforms, there is no consensus on its definition or how it manifests. Given the relationship among body image disturbance, social media, and negative emotional outcomes (Paxton et al., 2006; Pfund et al., 2020; Pikoos et al., 2021; Siegel, 2002), it is crucial for nurse practitioner's (NP) and other healthcare practitioners to recognize the occurrence of body image disturbance. Doing so will help to identify potential indicators and provide interventions in a timely manner.

\section{Background}

Presently, there is a lack of studies on body image disturbance in the context of online learning, especially in the adolescent population. Nonetheless, studies on traditional social media including Instagram, Facebook, and Snapchat, share common features with the online learning platforms. These features include selfie-viewing, distortion of digital images, and virtual 
engagement with peers. Given these similarities, studies of traditional social media were included to enrich the understanding of the concept in the context of online learning (Burnette et al., 2017; Mills et al., 2018; Pikoos et al., 2021; Vall-Roqué et al., 2021). In this paper, the term, online learning platform, refers to video-based online communicative systems currently used by adolescents for learning. Related terms, video chatting or video-conferencing platforms, refer to similar and/or identical video-based communicative systems that are being utilized for the purpose of work or post-secondary education.

A literature search was performed using databases pertaining to multiple disciplines, including Nursing, Psychology, Sociology and Medicine. The following three descriptors were used in the search: Body image disturbance, social media, and adolescents (Appendix A; Appendix B). Articles were reviewed for relevance and transferability. While effort was made to include studies on only adolescents, those that recruited both adolescents and adults were also included if the authors performed statistical analysis to account for the age variable. Articles that were published following the year 2004 were selected as this period marks the time when Facebook became publicly available and introduced the modern-day social media (Facebook, 2021). News articles were also included in the analysis to capture the present challenges faced by adolescents. Interestingly, while there was abundance of quantitative research, there was paucity of qualitative research studies. The final analysis included 13 empirical quantitative research articles, one empirical qualitative research article, and two newspaper articles (Appendix B; Appendix C).

Although generally used in reference to adolescents experiencing negative appearance related to social media, the search of the term 'body image disturbance' retrieved a wide variety of related terms, with no consensus on a definition of this concept. The uses of the term ranged from appearance dissatisfaction or concern (Pikoos et al., 2021; Seekis et al., 2020; Teran et al., 2020), selfie harm (Mills et al., 2018), body dissatisfaction (Burnette et al., 2017; Kleemans et al., 2018; McLean et al., 2015; Muñoz \& Ferguson, 2012; Pfund et al., 2020; Vall-Roqué et al., 2021), facial dissatisfaction (Wang et al., 2019; Yang et al., 2020), self-objectification (Fox et al., 2021; Yang et al., 2020), drive for thinness (McLean et al., 2015; Vall-Roqué et al., 2021), appearance comparisons (Burnette et al., 2017; Pfund et al., 2020; Yang et al., 2020), heightened awareness (Caltriders, 2021), self-esteem (Fox et al., 2021; Vall-Roqué et al., 2021), and zoom dysmorphia (Draksler, 2021; Pikoos et al., 2021). Further to the widely ranging definitions of body image disturbance, studies utilized a wide variety of outcomes measures, which calls for a need to harmonize the concept of body image disturbance in this newly emerged context.

The Canadian Paediatric Society (2019) recognizes the correlation between social media use and body image concerns, anxiety, and disordered eating in children and adolescents. The COVID-19 lockdown not only shifted in-school interactions to a virtual platform (Caltriders, 2021; Draksler, 2021; Pfund et al., 2020; Pikoos et al., 2021; Vall-Roqué et al., 2021), but also increased the overall use of social media sites such as Instagram, YouTube, TikTok, Twitter, and Facebook among adolescents (Vall-Roqué et al., 2021). Increased frequency of social media use was significantly correlated with body dissatisfaction, drive for thinness, and low self-esteem among adolescents and young adults (Vall-Roqué et al., 2021). 
Online learning platforms and traditional social media share features of perceptual disturbances. Recent news articles and research studies on zoom conferences and online learning platforms introduced the term '(zoom) dysmorphia', akin to 'snapchat dysmorphia' (Draksler, 2021; Pikoos et al., 2021), to highlight the perceptual disturbances that occur due to the limitations in the camera specifications (Bernetter et al., 2016; Cristel et al., 2020; Draksler, 2021; Pikoos et al., 2021; Třebický et al., 2016). Of those that used video chatting platforms over the past week, $37.4 \%$ reported noticing a new aspect of their appearance they disliked (Pikoos et al., 2021). These experiences are also shared in adolescent females using social media, as they report feeling "pretty in the mirror", but "ugly" on camera (Burnette et al., 2017). Viewing a mirrored reflection of a digitally distorted image of self and internalizing these images could have negative consequences for adolescent body image.

The notion of self-surveillance has surfaced in various articles of video chatting platforms and social media (Burnette et al., 2017; Mills et al., 2018; Pfund et al., 2020; Pikoos et al., 2021; Teran et al., 2020; Wang et al., 2019). Self-surveillance is grounded in the objectification theory, which states that individuals tend to internalize idealized appearances, leading to the awareness of their bodies such as facial attractiveness and symmetry. Once individuals internalize the idealized beauty, self-objectification can manifest as self-surveillance, a habitual monitoring of one's own appearance (Fredrickson \& Roberts, 1997). In adolescents and adults using video chatting platforms, approximately $27 \%$ reported primarily looking at their own face and body during their video chatting session (Pikoos et al., 2021). This finding is concerning as it has been suggested that the percentage of time spent looking at oneself, rather than simply the hours of video chatting, correlate with self -objectification (Pfund et al., 2020). Even as few as five minutes of self-focused attention can endorse decreased satisfaction with appearance, self-esteem, perceived attractiveness, and increased urge to change appearance (Barnier \& Collison, 2019). The awareness of one's own appearance elicits behavioural changes such as hiding self-view with $8 \%$ reported active avoidance of video calls with one of the reasons relating to appearance concerns (Pikoos et al., 2021). Similarly, adolescents frequently mentioned social media and social comparison in their appearance concerns and reduced confidence in their appearance (Burnette et al., 2017). Their self-surveillance manifested as investing time editing and selecting selfies even if their pictures were not being shared (Teran et al., 2020). These behaviours suggest that rather than the act of sharing selfies, it may be the act of self-surveillance and comparison with an internal standard of beauty that poses a negative influence on body image (Mills et al., 2018; Teran et al., 2020; Wang et al., 2019).

Appearance comparison occurs when adolescents compare their physical appearance to an external image of another individual. Often occurring in an upward direction towards the beauty ideal, a phenomenon well described by the social comparison theory (Festinger, 1954; Guo \& Wu, 2021; Jarman et al., 2021; Seekis et al., 2020); the theory posits that individuals strive to establish a position in their society by comparing themselves with others who are perceived to be similar on dimensions such as age and gender (Festinger, 1954; Seekis et al., 2020). Peer competition can be a stronger predictor of body dissatisfaction than symptoms of depression (Muñoz \& Ferguson, 2012). Adolescents and adults using video chatting platforms are constantly exposed to comparison, with 53\% of individuals reported focusing on both their own and others' face (Pfund et al., 2020; Pikoos et al., 2021). In adolescents using traditional social media, the tendency for appearance comparison in adolescents viewing unfamiliar peers 
on social media was higher than when viewing celebrities (Seekis et al., 2020), suggesting a potentially synergistic effect between social media and peers (Burnette et al., 2017; Kleemans et al., 2018). In both forms of social media, those who conveyed high tendency for appearance comparison expressed desire to change their appearance (Fardouly \& Vartanian, 2016; Pikoos et al., 2021) and engaged in behaviours such as hiding self-view and using features that enhanced their appearances (Pfund et al., 2020).

\section{Methods}

A concept analysis of body image disturbance among female adolescents using online learning platforms was conducted using Walker and Avant (2014) method. The eight steps of the Walker and Avant (2014) method are as follows: 1) Select a concept; 2) Determine the aims or purpose of analysis; 3) Identify all the uses of the concept possible; 4) Determine the defining attributes; 5) Identify model case; 6) Identify borderline, related, and illegitimate cases; 7) Identify antecedents and consequences; and 8) Define empirical referents. These steps were used to explore the concept.

\section{Concept Exploration}

\section{Current Uses of the Concept in the Literature}

The term "body image" is defined by the Merriam-Webster online dictionary (n.d.) as "a subjective picture of one's own physical appearance established both by self-observation and by noting reaction of others". In the healthcare disciplines, body image disturbance is defined by the NANDA-I nursing diagnosis as, "confusion in mental picture of one's physical self ... defined by characteristics such as the absence of body part, alteration in body function or structure, alteration in view of one's body" (Ladwig \& McKenzie, 2017, p. 167). The American Psychiatric Association (APA) describes body image disturbance as "a disturbance in the way in which body weight or shape is experienced" (APA, 2013, p. 242). This term is used in the Diagnostic and Statistical Manual of Mental Disorders 5 diagnosis of eating disorders such as anorexia nervosa, anorexia bulimia and eating disorders not otherwise specified (APA, 2013).

In the context of body dysmorphic disorder (BDD), body image disturbance has been primarily characterized as a debilitating preoccupation with self-perceived defect in appearance that ruminates as intrusive thoughts (Barnier \& Collison, 2019). It is defined as a "dynamic failure between the subjective experience of one's own body appearance with an objective appraisal of the body" (Espeset et al., 2011, p. 185).

In adults with previous treatment for cancer, body image disturbance is defined as the self-perception of and displeasure with a change in appearance, functional decline in an area, as well as the psychological distress that arise from these changes. It referred to the actual and sustained change in the appearance and function of their bodies (Rhoten, 2006).

In post bariatric surgery adult patients, body image disturbance was defined as the incongruencies in the experiences of the mind and the body manifested by changes in the body appearance that occurs faster than the mind can adapt. Individuals experienced negative 
influence on quality of life, arising from the excess skin after their weight loss, and inability to accept their new bodies diminished both social and physical functioning (Perdue et al., 2018).

\section{Determine the Defining Attributes}

The analysis of the different uses of the concept revealed three attributes on body image disturbance among adolescents using video chatting platforms and traditional social media. These included 1) feeling displeasure with the perceived appearance while using video streamed platforms; 2) Self-surveillance of one's appearance; and 3) upward comparison of one's appearance with others and experiencing feelings of inadequacy.

\section{Feeling displeasure with the perceived appearance while using video streamed platforms}

While body image disturbances can be experienced without digital images, for this phenomenon to manifest specifically in the context of online learning, the platform must be such that it has live-streamed videos that mirror digitally distorted images (Burnette et al., 2017; Draksler, 2021; Pikoos et al., 2021). Furthermore, they must feel displeasure with the perceived appearance even when individuals are no longer able to view their self image. While others might not notice the difference between the real and these distorted images, adolescents must feel displeasure with the distorted image of oneself (Burnette et al., 2017; Pfund et al., 2020; Pikoos et al., 2021). If adolescents do not recognize this change or do not feel displeasure with the perceived appearance, then body image disturbance does not occur.

This attribute is distinguished from BDD by the presence of a true externally distorted image of oneself, rather than an altered cognitive psychological process in the absence of this external change (Espeset et al., 2011). It is also distinguished from post-bariatric surgery patients and cancer survivors by absence of a physical change in one's body (Perdue et al., 2018; Rhoten, 2016), but rather a digitally disturbed image of oneself.

\section{Self-surveillance of one's appearance}

For adolescents to experience body image disturbance, adolescents must exhibit vigilant monitoring of one's outward body appearance or self-surveillance, either by monitoring their own appearance (Caltriders, 2021; McLean et al., 2015) or engaging in activities to enhance their appearance even when these images are not being shared (Fox et al., 2021; Mills et al., 2018; Teran et al., 2020; Wang et al., 2019). Self-surveillance can also manifest as behavioural changes, such as adjusting the position of their camera to show the best angles on video (Burnette et al., 2017; Pfund et al., 2020; Pikoos et al., 2021). While adolescents still experience negative feelings and anxiety when editing their appearance (Mills et al., 2018; Teran et al., 2020), this behaviour may help them feel some degree of control over how they are viewed (Mills et al., 2018).

While this attribute is very similar to BDD, the difference is that the surveillance behaviour stops once the external triggers are halted, as opposed to the BDD, where their urge to self surveillance ruminates and manifests as intrusive thoughts (Barnier \& Collison, 2019). This attribute also differs from body image disturbance from post-bariatric surgery (Perdue et al., 2018) and cancer survivors (Rhoten, 2016) as these populations have actual physical or 
functional changes in their body. Their body disturbance is manifested rather by the grievance of their old selves, and unfamiliarity with the undesired changes.

\section{Upward comparison of one's appearance with others and experiencing feelings of inadequacy}

Body image disturbance in adolescents occur when they compare their appearances with other individuals whose appearance lies closer to the societal beauty ideals (Pfund et al., 2020; Pikoos et al., 2021; Seekis et al., 2020). This attribute does not occur when adolescents compare themselves with others whom the adolescent believes is downwards from the socio-culturallydefined beauty ideals (Yang et al., 2020). The degree to which this attribute manifests in the adolescent varies, where comparison with unfamiliar peers on social media has a greater effect than viewing celebrities (Burnette et al., 2017; Kleemans et al., 2018; Muñoz \& Ferguson, 2012; Seekis et al., 2020).

This attribute is distinguished from BDD in that BDD - it is characterized by preoccupation with images that are distorted by their own cognitive-perceptual process. The comparison is made upwards with a perceptually disturbed ideal appearance of themselves (Espeset et al., 2011). Body image disturbance in post-bariatric surgery and cancer survivors occur due to comparison with their previous selves or function (Perdue et al., 2018; Rhoten, 2016), and not due to comparison with their peers.

\section{Constructed Cases}

\section{Model Case}

Clara is a 15-year-old girl who currently attends her classes through Zoom. She hates having her webcam on as she feels like her nose looks abnormally large compared to the other girls in class. Today, she catches herself monitoring her own face again, as she tries to adjust the lighting and the camera angle, hoping that it would improve her appearance. Not satisfied with her appearance, she turns off the video, while intermittently monitoring her classmates' appearances on video. After her class, she practices taking selfies, trying to figure out the best angle for her appearance. She takes multiple selfies before finding the perfect angle that improves the appearance of her nose.

A model case contains all the defining attributes of the concept (Walker et al., 2014). This is a model case as it contains the attributes of: Feeling displeasure with the perceived appearance while using video streamed platforms; conducting self-surveillance of one's appearance; and comparing one's appearance to those perceived as closer to the ideals and experiencing feelings of inadequacy. Clara views a digitally distorted reflection of herself and experiences negative emotions towards her appearance. She compares herself with other classmates whom she believes look better than her. She surveillances her own appearance on Zoom and becomes pre-occupied with the way she looks. Even when she turns off her video, she continues to feel self-conscious about her appearance and intermittently monitors other classmates' appearance. After her class, she takes multiple selfies at home to have the perfect image even though she does not share it. 


\section{Borderline Case}

Mandy is a 15-year-old girl. She enjoys taking selfies and usually invests a lot of time editing them. However, she does not post them on her social media account. Usually, she also has a YouTube video playing on the side of her favourite influencer and tries to imitate her pose.

A borderline case contains most of the defining attributes of the concept but not all of them (Walker \& Avant, 2014). This case exemplifies some defining attributes: Viewing a digitally distorted image of oneself; conducting self-surveillance of one's appearance; and comparing one's appearance to those perceived as closer to the ideals and experiencing feelings of inadequacy. Mandy may view a digitally distorted reflection of herself. However, note that she does not necessarily feel displeasure associated with the distorted images, which is one of the key attributes that endorses body image disturbance. She exhibits self-surveillance and appearance preoccupation, as demonstrated by taking multiple selfies and investing time on editing them without sharing them. She also engages in upward comparison by trying to emulate the social media influencer.

\section{Related Case}

Jody is a 15-year-old female, who is currently using zoom for her online high school. She has been feeling exceptionally down lately and Zoom seems to amplify the bags under her eyes. She compares herself to her classmates, who look refreshed, carefree, and happy. She has been struggling with depressive symptoms ever since her mom had passed away 6 months ago. Not wanting to be seen, she dims the light in her room and adjusts the webcam such that only a portion of her face is seen.

A related case is similar to the concept being studied, and may include some of the attributes that can be mistaken for the defining attributes of the concept (Walker \& Avant, 2014). This case is a related case because it contains the attributes of: Viewing a digitally distorted image of oneself; conducts self-surveillance of one's appearance; and compares one's appearance to those perceived as closer to the ideal. Jody views a distorted image of herself on Zoom but does not internalize the defined standard of beauty. While she evaluates her own appearance and compares it with those of her classmates, this action is not conducted in a manner that compares herself to the beauty ideals. She compares herself to other students, but this is unrelated to beauty ideals. Rather, it compares the grievance of her mom's passing to other classmates who appear carefree in their seemingly ideal family life. The attributes present in this scenario can be mistaken for the defining attributes of the concept of body image disturbance, but in no way is related to body image disturbance.

\section{Illegitimate Case}

An illegitimate case uses the concept term out of context (Walker \& Avant, 2014). Skipping stones on a large body of water disturbed a calm image of nearby cottages is an example of an illegitimate case. 


\section{Antecedents and Consequences}

Antecedents are events that must occur prior to the occurrence of a concept (Walker \& Avant, 2014). For body image disturbance to occur in this context, an individual must be an adolescent and using video-based online learning platforms that allow for potential interaction with others who can be identified as peers. Secondly, the individuals' image of themselves must be streamed live to their peers, with the potential to be mirrored back to them. The third antecedent is that adolescents need to have a peer with whom they perform upwards comparison.

Consequences are events that occur due to the occurrence of the concept (outcomes) (Walker \& Avant, 2014). The main consequence of body image disturbance is alterations in mood and lowered self esteem (Fox et al., 2021; Mills et al., 2018; Vall-Roqué et al., 2021). This can take in the form of avoiding taking photos or self views, adjusting their camera, hiding selfviews, and applying filters to alter their image (Castelli \& Savary, 2021; Gherhes et al., 2021; Pikoos et al., 2021). Other potential events include utilizing avatars that are closer to the adolescents' beauty ideals or those that are simply "fun" and maintain their relative anonymity (de Gomes de Siqueira et al., 2021; Jun, 2021; Loewen et al., 2021). Those adolescents not employing avatars may choose to turn off their cameras and be asked repeatedly to turn on their videos.

\section{Empirical Referents}

Empirical referents are categories of actual phenomena that by their presence demonstrate the occurrence of the concept itself. Empirical referents are not tools to measure the concept, but the means by which you can recognize or measure the defining characteristics. The empirical referents relate directly to the defining attributes, not the entire concept itself (Walker \& Avant, 2014). These may manifest as being concerned with aspect of body not recognized by others; preoccupation with their appearance, selfie editing, or video avoidance; and looking at images of others and expressing desire to change the appearance of their face, hair or skin (Fardouly \& Vartanian, 2016; Pikoos et al., 2021). Adolescents who are concerned with their body image may subsequently experience psychological distress while engaging in online learning.

\section{Concept Definition}

For female adolescents using online learning platforms, body image disturbance can be experienced as primarily perceptual, cognitive, and behavioural changes. These include the displeasure with one's distorted appearance felt during live video streamed online learning platforms, self-surveillance of one's own appearance, and comparison of oneself with others who lies closer to the beauty ideals. These negative feelings about oneself leads to behavioural changes of selfie avoidance, video manipulation, and camera angle adjustment.

\section{Discussion}

This concept analysis establishes the defining attributes of body image disturbance among female adolescents in the context of online learning platforms. The nature of these 
platforms allows direct comparison of digitally distorted images of oneself with others in real time, upon which female adolescents may start to experience disturbances in their body image. A standardized understanding of this concept will allow NPs and other healthcare providers to recognize its occurrence and intervene appropriately to support the adolescent and work with the teachers create a safe and supportive learning environment. The standardized concept is also relevant to clinical practice, education, research, and policy and leadership.

From a clinical standpoint, NPs can operationalize the definition of body image disturbance to screen for and recognize the occurrence of the event. While there is currently no validated tool in the detection of body image disturbance in this context, this definition can be operationalized with questionnaires that pertain to the defining attributes of the concept. The Dysmorphic Concern Questionnaire (DCQ) measures the concerns with physical appearance and beliefs about individual's body (Pikoos et al., 2021); the general attractiveness internalization subscale of the Sociocultural Attitudes Towards Appearance Questionnaire-4Revised (SATAQ-4R), which assesses a general desire to be attractive (Wang et al., 2019); and the physical appearance comparison scale, which addresses questions related to the video chatting appearance comparison (Pfund et al., 2020). A survey of European student and practising healthcare providers revealed that individuals varied in their ability to recognize and address their patients' appearance concerns (Williamson et al., 2018). There appears to be similar concerns in Canada with respect to NP mental health education (Creamer \& Austin, 2017). As such, these tools can be a useful tool for NPs, as well as other healthcare providers that interact with adolescents. Furthermore, these attributes of body image disturbance can be shared with adolescents, parents, and teachers to educate them on ways to identify potential indicators of its occurrence.

COVID-19 continues to disrupt traditional educational experience, with components of online learning extending into the upcoming school year (Government of Ontario, 2021). Given the continued disruption, ongoing use of online learning platforms in adolescents must involve change. Body image disturbance can lead to not only social avoidance, drive for thinness, and lower self esteem (Guo \& Wu, 2021; Pikoos et al., 2021; Seekis et al., 2020; Teran et al., 2020), but also pose as a risk factor to psychological disorders such as anorexia nervosa (APA, 2013, p. 338). NPs can educate their clients about cameras, such as webcams and phones, distorting facial appearances as a reality (Cristel et al., 2020; Ward et al., 2018). Increased use of social media and video filters seems to endorse higher levels of anxiety and self-scrutiny among postsecondary students (Silence et al., 2021). Furthermore, each encounter with adolescents can be used as an educational opportunity to discuss the unintended secondary effects of using extensive touch-up filters during video conferences (Silence et al., 2021). In a study of post-secondary students, video filter users displayed higher levels of anxiety with return to in-person school compared to non-filter users ( $85 \%$ vs. 58\%, respectively) (Silence et al., 2021). Overall, adolescents should be counselled to be mindful of unintended consequences while engaging in video based online learning platforms.

Similarly, NPs can also educate teachers on refraining mandatory video-use among adolescents as well as hiding self-view features and providing an option to use avatars. Not only are the cameras digitally altering images, but staring at one's own reflection can lead to further worsening of one's perception of their appearance (Barnier \& Collison, 2019). Even if the 
images are not shared, there seems to be continued risk of self-surveillance (Teran et al., 2020). While the benefits of using avatars are not entirely known, current literature seems to suggest that it can maintain relative anonymity, give students opportunity to have fun, reinforce the experience of being in the virtual world (de Gomes de Siqueira et al., 2021), and lead to higher rate of class participation (Jun, 2021). Whether the avatar selection choice relates to adolescents' psychological well being is unknown in the context of online learning; studies of avatar selection for video games among post-secondary students suggest that the avatar can predict individuals' psychological well-being and self discrepancy. There may be larger perceived discrepancy in those who selected ideal versus realistic avatars (Loewen et al., 2021). Therefore, it may be worthwhile for future research to explore avatar selection choices as an indicator of psychological well-being.

In the context of virtual medicine, clients, especially those with eating disorders, discussing their health concerns while viewing themselves on the screen may pose an added layer of distress and even potentially hinder access to receiving health care. In such scenarios, minimizing their on-screen appearance or hiding clients' self-view appearance may alleviate some of the in-session distress (Matheson et al., 2020). Alternatively, NPs can utilize this technology as an opportunity for exposure therapy for gradual acceptance of viewing their selves on screen (Matheson et al., 2020). When such measures during virtual appointments are unsuccessful and pose as barrier to accessing healthcare, NPs can also advocate for in-person appointments, provided that it aligns with the current pandemic and organizational guidelines. Further research could seek ways to minimize potential detrimental effects of virtual medicine on adolescent body image. The working definition of body image disturbance can be used in the developmental phase of virtual medicine platforms to identify if these forms of healthcare are safe for client use.

\section{Conclusion}

While the issue of body image and social media was present before the COVID-19 pandemic, the extensive exposure to online learning platforms among adolescents is a newly emerged phenomenon, heightened by the COVID-19 pandemic. While there are limited studies on this phenomenon, NPs can use the scenarios presented in this study, along with examples of questionnaires, to recognize and delineate the occurrence of body image disturbance versus a borderline or a related case. Not only does this definition serve purpose directly in clinical practice, but also in research; NPs can further utilize the definition to develop a validated instrument that can accurately measure the occurrence of this concept; NPs can also be consulted in the research and development of online learning activities that are less psychologically harmful to female adolescents' sense of body image. Finally, the definition presented in this concept analysis will not only enable NPs, but also adolescents, parents, teachers, and other healthcare providers to recognize the occurrence of body image disturbance in female adolescents using virtual chatting platforms. 


\section{References}

American Psychiatric Association. (2013). Diagnostic and statistical manual of mental disorders (5th ed.). Washington, DC: American Psychiatric Association.

Barnier, E. M., \& Collison, J. (2019). Experimental induction of self-focused attention via mirror gazing: Effects on body image, appraisals, body-focused shame, and self-esteem. Body Image, 30, 150-158. https://doi.org/10.1016/j.bodyim.2019.07.003

Burnette, C. B., Kwitowski, M. A., \& Mazzeo, S. E. (2017). “I don't need people to tell me I'm pretty on social media:" A qualitative study of social media and body image in early adolescent girls. Body Image, 23, 114-125. https://doi.org/10.1016/j.bodyim.2017.09.001

Caltriders, M. (2021, Feb 26). Negative body image concerns increase as pandemic creates 'virtual world'. Retrieved June 17, 2021 from abc57 news. https://www.abc57.com/news/negative-body-image-concerns-increase-as-pandemiccreates-virtual-world

Castelli, F. R., \& Sarvary, M. A. (2021). Why students do not turn on their video cameras during online classes and an equitable and inclusive plan to encourage them to do so. Ecology and Evolution, 11(8), 3565-3576. https://doi.org/10.1002/ece3.7123

Canadian Paediatric Society, Digital Health Task Force, Ottawa, Ontario. (2019). Digital media: Promoting healthy screen use in school-aged children and adolescents. Paediatrics \& Child Health, 24(6), 402-408. https://doi.org/10.1093/pch/pxz095

Creamer, A. M., \& Austin, W. (2017). Canadian nurse practitioner core competencies identified: An opportunity to build mental health and illness skills and knowledge. Journal for Nurse Practitioners, 13(5), e231-e236. https://doi.org/10.1016/j.nurpra.2016.12.017

Cristel, R. T., Demesh, D., \& Dayan, S. H. (2020). Video conferencing impact on facial appearance: Looking beyond the COVID-19 pandemic. Facial Plastic Surgery \& Aesthetic Medicine, 22(4), 238-239. https://doi.org/10.1089/fpsam.2020.0279

D2L. (2020, March 25). Virtual classroom for Brightspace. [Video]. YouTube. https://www.youtube.com/watch?v=mvuhQoGU3_o

Draksler, K. (2021, Jan 22). Video calls causing appearance insecurities, so-called 'zoom dysmorphia'. Retrieved on June 17, 2021, from KTSM. https://www.ktsm.com/health2/video-calls-causing-appearance-insecurities-so-called-zoom-dysmorphia/

Elkind, D. (1967). Egocentrism in adolescence. Child development, 1025-1034. https://doi.org/10.2307/1127100

Espeset, E. M., Nordbø, R. H., Gulliksen, K. S., Skårderud, F., Geller, J., \& Holte, A. (2011). The concept of body image disturbance in anorexia nervosa: An empirical inquiry 
utilizing patients' subjective experiences. Eating Disorders, 19(2), 175-193. DOI: 10.1080/10640266.2011.551635

Fardouly, J., \& Vartanian, L. R. (2016). Social media and body image concerns: Current research and future directions. Current Opinion in Psychology, 9, 1-5. https://doi.org/10.1016/j.copsyc.2015.09.005

Facebook. (2021). Who we are: Company info. Retrieved on June 10, 2021, from https://about.facebook.com/company-info/

Festinger, L. (1954). A theory of social comparison processes. Human Relations, 7(2), 117-140. https://doi.org/10.1177/001872675400700202.

Fox, J., Vendemia, M. A., Smith, M. A., \& Brehm, N. R. (2021). Effects of taking selfies on women's self-objectification, mood, self-esteem, and social aggression toward female peers. Body Image, 36, 193-200. https://doi.org/10.1016/j.bodyim.2020.11.011

Fredrickson, B. L., \& Roberts, T.-A. (1997). Objectification theory: Toward understanding women's lived experiences and mental health risks. Psychology of Women Quarterly, 21, 173-206. http://dx.doi.org/10.1111/j.1471-6402.1997.tb00108.x

Gherhes, V., Simon.S., \& Para, I. (2021). Analysing students' reasons for keeping webcams on or off during online classes. Sustainability, 13, 3203. https://doi.org//10.3390/su13063203

Government of Ontario. (2020, July 28). Guide to reopening Ontario's schools. https://www.ontario.ca/page/guide-reopening-ontarios-schools

Government of Ontario. (2021, August 13). Covid-19: Health, safety and operational guidance for schools (2021-2022). https://www.ontario.ca/document/covid-19-health-safety-andoperational-guidance-schools-2021-2022

Guo, Q., \& Wu, M. (2021). The relationship between self-objectification and social avoidance among Chinese middle adolescent girls: The mediating role of appearance comparison and self-esteem. Current Psychology, 1-9. https://doi.org/10.1007/s12144-021-01705-8

Jarman, H. K., Marques, M. D., McLean, S. A., Slater, A., \& Paxton, S. J. (2021). Social media, body satisfaction and well-being among adolescents: A mediation model of appearanceideal internalization and comparison. Body Image, 36, 139-148.

https://doi.org/10.1016/j.bodyim.2020.11.005

Joanna Briggs Institute (n.d.) Critical appraisal tools. Retrieved June 8, 2021 from https://jbi.global/critical-appraisal-tools

Hogan, M. J. \& Strasburger, V. C. (2008). Body image, eating disorders, and the media. Adolescent Medicine: State of the Art Reviews, 19(3):521-46, x-xi. PMID: 19227390. 
Kleemans, M., Daalmans, S., Carbaat, I., \& Anschütz, D. (2018). Picture perfect: The direct effect of manipulated Instagram photos on body image in adolescent girls. Media Psychology, 21(1), 93-110. https://doi.org/10.1080/15213269.2016.1257392.

Ladwig, G. B., \& McKenzie, M. (2017). Disturbed Body Image. In B. J. Ackley, G. B. Ladwig \& M. B. F. Makic (Eds). Nursing Diagnosis Handbook: An evidence-based guide to planning care (11th ed., pp. 167-171). St. Louis, Missouri: Elsevier

Merriam-Webster. (n.d.). Body-image. In Merriam-Webster.com dictionary. Retrieved June 14, 2021, from https://www.merriam-webster.com/medical/body\%20image

Mills, J. S., Musto, S., Williams, L., \& Tiggemann, M. (2018). "Selfie" harm: Effects on mood and body image in young women. Body Image, 27, 86-92. https://doi.org/10.1016/j.bodyim.2018.08.007

Muñoz, M. E., \& Ferguson, C. J. (2012). Body dissatisfaction correlates with inter-peer competitiveness, not media exposure: A brief report. Journal of Social and Clinical Psychology, 31(4), 383-392. https://doi.org/10.1521/jscp.2012.31.4.383

Paglinawan, D. (2020, Jul 5). Virtual learning expected to continue in many provinces due to coronavirus. Retrieved December 4, 2021 from cbc news. https://www.cbc.ca/news/science/schools-virtual-learning-privacy-1.5615999

Paxton, S. J, Neumark-Sztainer, D., Hannan, P.J., \& Eisenberg ME. (2006). Body dissatisfaction prospectively predicts depressed mood and low self-esteem in adolescent girls and boys. Journal of Clinical Child Adolescent Psychology. 35(4):539-549. https://doi.org/10.1027/s15374424ijccp3504_5

Perdue, T. O., Schreier, A., Neil, J., Carels, R., \& Swanson, M. (2018). A concept analysis of disturbed body image in bariatric surgery patients. International Journal of Nursing Knowledge, 31(1), 74-81. https://doi.org/10.1111/2047-3095.12220

Pfund, G. N., Hill, P. L., \& Harriger, J. (2020). Video chatting and appearance satisfaction during COVID-19: Appearance comparisons and self-objectification as moderators. International Journal of Eating Disorders, 53(12), 2038-2043. https://doi.org/10.1002/eat.23393

Pikoos, T., Buzwell, S., Sharp, G., \& Rossell, S. (2021). The 'Zoom Effect': Exploring the impact of video-calling on appearance dissatisfaction and interest in aesthetic treatment during the COVID-19 pandemic. Aesthetic Surgery Journal, 41(2), 2066-2075. http://doi.org/10.1093/asj/sjab257

Rhoten, B. A. (2016). Body image disturbance in adults treated for cancer- A concept analysis. Journal of Advanced Nursing, 72(5), 1001-1011. https://doi.org/10.1111/jan.12892 
Seekis, V., Bradley, G. L., \& Duffy, A. L. (2020). Appearance-related social networking sites and body image in young women: Testing an objectification-social comparison model. Psychology of Women Quarterly, 44(3), 377-392. https://doi.org/10.1177/0361684320920826

Siegel, J. M. (2002). Body image change and adolescent depressive symptoms. Journal of Adolescent Research, 17(1):27-41. https://doi.org/10.1177/0743558402171002

Silence, C., Rice, S. M., Pollock, S., Lubov, J. E., Oyesiku, L. O., Ganeshram, S., ... \& Kourosh, A. S. (2021). Life after lockdown: Zooming out on perceptions in the postvideoconferencing era. International Journal of Women's Dermatology, 2021. https://doi.org/10.1016/j.ijwd.2021.08.009

Teran, L., Yan, K., \& Aubrey, J. S. (2020). "But first let me take a selfie": US adolescent girls' selfie activities, self-objectification, imaginary audience beliefs, and appearance concerns. Journal of Children and Media, 14(3), 343-360. https://doi.org/10.1080/17482798.2019.1697319

Třebický, V., Fialová, J., Kleisner, K., Havlíček, J. (2016). Focal length affects depicted shape and perception of facial images. Public Library of Science One. 11(2):e0149313. https://doi.org/10.1371/journal.pone.0149313

United Nations Educational, Scientific and Cultural Organization. (2020). COVID-19 educational disruption and response. https://en. unesco.org/themes/educationemergencies/coronavirus-schoolclosures

Vall-Roqué, H., Andrés, A., \& Saldaña, C. (2021). The impact of COVID-19 lockdown on social network sites use, body image disturbances and self-esteem among adolescent and young women. Progress in Neuro-Psychopharmacology and Biological Psychiatry, 110, 110293. https://doi.org/10.1016/j.pnpbp.2021.110293

Walker, L. O., \& Avant, K. C. (2014). Strategies for Theory Construction in Nursing, 5th ed. Essex, England: Pearson Education Limited.

Wang, Y., Fardouly, J., Vartanian, L. R., \& Lei, L. (2019). Selfie-viewing and facial dissatisfaction among Chinese adolescents: A moderated mediation model of general attractiveness internalization and body appreciation. Body Image, 30, 35-43. https://doi.org/10.1016/j.bodyim.2019.05.001

Ward, B., Ward, M., Fried, O., \& Paskhover, B. (2018). Nasal distortion in short-distance photographs: The selfie effect. The Journal of the American Medical Association Facial Plastic Surgery, 20(4), 333-335. https://doi.org/10.1001/jamafacial.2018.0009

World Health Organization. (2020, March 12). WHO Director-General's opening remarks at the Mission briefing on COVID-19. https://www.who.int/dg/speeches/ detail/who-directorgeneral-s-opening-remarks-at-the-missionbriefing-on-covid-19 
Williamson, H., Antonelli, P., Bringsén, Å., Davies, G., Dèttore, D., Harcourt, D., ... \& Persson, M. (2018). Supporting patients with body dissatisfaction: A survey of the experiences and training needs of European multi-disciplinary healthcare professionals. Nursing Plus Open, 4, 8-13. https://doi.org/10.1016/j.npls.2018.05.001

Yang, J., Fardouly, J., Wang, Y., \& Shi, W. (2020). Selfie-viewing and facial dissatisfaction among emerging adults: A moderated mediation model of appearance comparisons and self-objectification. International Journal of Environmental Research and Public Health, 17(2), 672. https://doi.org/10.3390/ijerph17020672 


\section{Inclusion/ Exclusion criteria}

\section{Appendix A}

Inclusion criteria

Date: Articles published after 2004 (introduction of first modern day social media - Facebook) Language: English language

Population of interest: All literature which evaluates adolescent perception, feelings, behaviour with respect to social media

- Articles where adolescent and younger adults were included as a single sample population were included in this paper

- Adolescent: Defined as 13 - 18 years of age, as per CINAHL

Gender: Female gender

Type of publication: Peer reviewed journal articles from all disciplines of all study designs

- Grey literature (News articles, Blogs)

- Professional opinion 
Search Strategy

Appendix B

\begin{tabular}{|c|c|c|c|c|}
\hline Database & Search terms & $\begin{array}{l}\text { Total \# of } \\
\text { retrieved } \\
\text { articles }\end{array}$ & $\begin{array}{l}\text { Total \# } \\
\text { included in } \\
\text { the final } \\
\text { analysis } \\
\end{array}$ & \\
\hline CINAHL & $\begin{array}{l}\text { Body image or body } \\
\text { dissatisfaction or body image } \\
\text { disturbance or facial } \\
\text { dissatisfaction AND social media } \\
\text { or covid-19 AND adolescent }\end{array}$ & 96 & 4 & 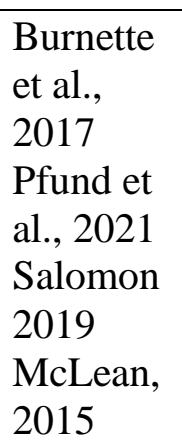 \\
\hline PsychINFO & $\begin{array}{l}\text { ((body image disturbance) OR } \\
\text { (body dissatisfaction)) AND } \\
\text { ("social media" or "online } \\
\text { learning" or "distance learning" } \\
\text { or "virtual learning") AND } \\
\text { "adolescence" }\end{array}$ & 133 & 3 & $\begin{array}{l}\text { Muñoz et } \\
\text { al., } 2012 \\
\text { Seekis et } \\
\text { al., } 2020 \\
\text { Kleemans } \\
\text { et al, } 2018\end{array}$ \\
\hline $\begin{array}{l}\text { ProQuest - } \\
\text { Sociology } \\
\text { Collection }\end{array}$ & $\begin{array}{l}\text { ((body image disturbance) OR } \\
\text { body dissatisfaction)) AND } \\
\text { ("social media" or "online } \\
\text { learning" or "distance learning" } \\
\text { or "virtual learning") AND } \\
\text { "adolescence" }\end{array}$ & 13 & 0 & \\
\hline Ovid - EMBASE & $\begin{array}{l}\text { ("Body image disturbance" OR } \\
\text { "self concept" OR "body } \\
\text { dissatisfaction" OR "self- } \\
\text { objectification") AND ("online- } \\
\text { learning" or "e-learning" or } \\
\text { "virtual learning" or "online } \\
\text { school" or "social media" AND } \\
\text { "adolescence" }\end{array}$ & 96 & 2 & $\begin{array}{l}\text { Vall- } \\
\text { Roqué } \\
2021 \\
\text { Fox et al., } \\
2021\end{array}$ \\
\hline Google & $\begin{array}{l}\text { COVID19 adolescent body image } \\
\text { news }\end{array}$ & & 2 & $\begin{array}{l}\text { Caltriders, } \\
2021 \\
\text { Draksler, } \\
2021\end{array}$ \\
\hline $\begin{array}{l}\text { Related articles/ } \\
\text { reference list }\end{array}$ & & & 5 & $\begin{array}{l}\text { Teran et } \\
\text { al., } 2020 \\
\text { Mills et } \\
\text { al., } 2018 \\
\text { Yang et } \\
\text { al., } 2020\end{array}$ \\
\hline
\end{tabular}


Nurse Practitioner Open Journal

DOI 10.28984/npoj.vi2.361

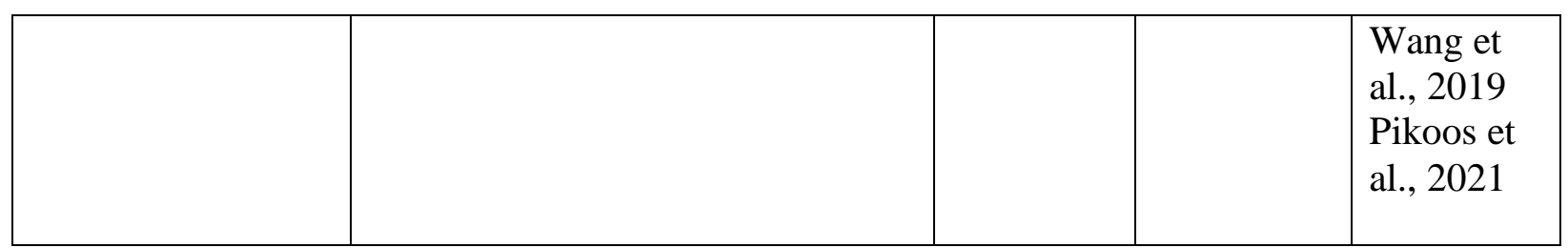




\section{Appendix C}

Caltriders, 2021

1. Is the source of the opinion clearly identified?

2. Does the source of opinion have standing in the field of expertise?

3. Are the interests of the relevant population the central focus of the opinion?

4. Is the stated position the result of an analytical process, and is there logic in the opinion expressed?

5. Is there reference to the extant literature?

6. Is any incongruence with the literature/sources logically defended?

$$
\text { appearance }
$$

Yes, author of the newspaper article and the
family psychologist interviewed is identified

yes

Yes, seeks to understand the impact of zoom use on teenagers sense of control and

Yes, the main point is that zoom classrooms pose a mental health risk to students

Yes, but not those that directly pertain to zoom and appearance

Yes, but only mentioned the study of body image and mental health
Draksler, 2021

Yes, author of the newspaper article and the psychiatrist interviewed is identified

yes

Yes, seeks to understand the impact of zoom use on students perception of their appearance

Yes, the main point is that zoom classrooms pose a mental health risk to students

\section{Reference made to a study but}

link/information not available

Yes, but only mentioned a study of dermatology that saw similar trends in appearance insecurity

Figure C1. Text and opinion papers (Joanna Briggs Institute, n.d.)

Were the criteria for inclusion in the sample clearly defined?

Were the study subjects and the setting described in detail?

Vall-Roque et al 2021
All Spanish women ages 14-
35 yo
Described place of residence,
employment \& relationship
status, whether or not
contracted covid or loved
one infected/deceased from
covid 19, and own space to
relax at home
Family income status, siblings
were not explored which

Teran et al., 2020

All girls 14-17 yo recruited through a private survey firm through their parents

Females and males from college between 17-22 years of age
Muñoz \& Ferguson, 2012

Female students aged 14-34 yo. But doesn't describe the distribution of age

Could benefit from more info about SES, relationship with parents, where the survey was completed etc.
Study setting was not described and the demographic info was not published
No. Perhaps ne
regarding home

environment, \# computers at

home etc 
Was the exposure measured in a valid and reliable way?

Were objective, standard criteria used for measurement of the condition?

Were confounding factors identified?

Were strategies to deal with confounding factors stated?

Were the outcomes measured in a valid and reliable way? could be important

Cronbach's alpha $>0.85$ for all

yes

Yes. Several identified

Assessed BMI and social media use, race used as dichotomous variable due to

restricted diversity

19 , current relationship

status, home environment

Selfie activities - sharing frequency, editing, and

Likert scale self report of SNS

site use frequency

Eating disorders inventory

(EDI-3)

Rosenberg self esteem scale

(RSES)

Cronbach's alpha $>0.85$

or the selfie

shortening of question

prevent participant fatigue investment (5 pt scoring system); self objectification (self-objectification beliefs and behaviours scale); appearance concerns (social appearance anxiety scale)
Cronbach's alpha $>0.84$

for all

Coefficient alpha $>0.70$ imaginary audience beliefs (New imaginary audience scale); social media duration
See \#7

See \#7 yes

Assessed BMI, perception of parental affection, media exposure, depression, verbal abuse, female competition

Selfie-viewing ( 3 item questions with $1 \times 6 \mathrm{pt}$ and $2 \times 7 p t$ scale, standardized); appearance comparison (physical appearance comparison scale - PACS); self objectification questionnaire (SOQ);

Facial dissatisfaction

(Facial appearance

concerns - FAC subscale of the Negative physical self scale (NPSS)
Body image dissatisfaction Body esteem scale for adolescents and adults BESAA); satisfaction with life scale (SLS) 
Was appropriate statistical analysis used?

One way ANOVA, Heges'g Wilcoxon signed rank test Chi-square test of independence
Yes, used Analysis of

Moment Structures (AMOS)

program with model fit

based on statistically

calculated criteria. Used $\mathrm{X}^{2}$

to investigate the fit of the

model compare to alternate model

Figure C2. Cross sectional studies (Joanna Briggs Institute, n.d.)

\section{Cross Sectional Studies}

Were the criteria for inclusion in the sample clearly defined?

Were the study subjects and the setting described in detail?

Was the exposure measured in a valid and reliable way?
Seekis et al., 2020

Female Australian undergraduate

students enrolled in the $1^{\text {st }}$ year

psych course

Yes, participants to complete questionnaire on secure website

Exploratory factor analysis using principal axis factoring and oblique rotation were conducted to establish structural validity for appearance related SNS use.

Parallel analysis, velicer's minimum average partial test (MAP). Exclusion of factors with fewer than 3 items, a factor loading cut off, percentage of total variance explained by each factor
Wang et al., 2019 Boys and girls from middle school and high school in China

Unsure where and how the survey was delivered

Selfie viewing as used by other studies. Cronbach alpha 0.65

Selfie viewing as used
by other studies.
Cronbach alpha 0.65

$\begin{array}{ll}\text { PROCESS macro model in } & \begin{array}{l}\text { Pearson's correlation } \\ \text { coefficient }\end{array} \\ \text { SPSS } & P<0.001\end{array}$

PROCESS macro model in

$\mathrm{P}<0.001$
McLean et al., 2015 Female gr7 students from secondary school (mean age 13.13 yo)

Yes, online survey completed during class time and was supervised by a researcher and class teacher

Demographic info, media exposure (6-item version of a media use questionnaire), photo activity (self image taking frequency, self image sharing, photo investment, photo manipulation)

Cronbach $\mathrm{a}=0.72$, coefficient $>0.82$

\section{Pikoos et al., 2021}

Any adults 18 yo and over currently residing in Australia

Online survey done at home

Demographic info, video call usage (unknown validity of measurement) 
Were objective, standard criteria used for measurement of the condition?

Were confounding factors identified?

Were strategies to deal with confounding factors stated?

Were the outcomes measured in a valid and reliable way?

Was appropriate statistical analysis used?
Upward physical appearance comparison scale $(a=0.95)$, body surveillance subscale from the objectified body consciousness scale $(a=0.89)$, social appearance anxiety scale $(a=0.94)$, drive for thinness subscale from the eating disorders inventory $3(a=0.83$, body dissatisfaction subscale from the eating disorders inventory $3(a=0.94)$

Yes, evaluated type of SNS, BMI, race, attention. But not SES,

family dynamics

$$
\text { Y }
$$

Yes, used data analysis to exclude participants who failed attention checks

Yes (see \#4)

SPSS
General attractivenes subscale of the sociocultural attitudes towards appearance questionnaire, revised (SATAQ-4R), body appreciation scale-2 (BAS-2), facial appearance concern subscale of the negative physical self scale (Cronbach a > 0.80)

Assesses the type of selfies reviewed and BMI but not other factors like SES, family dynamics etc.
Missing data was handled by pairwise deletion

Yes (see \#4)

PROCESS macro

SPSS 10-item body

dissatisfaction subscale of

the eating disorders inventory 3 (0.89); dietary restraint (10-item Dutch eating behaviour questionnaire restraint subscale. 0.94);

internalization of the thin ideal (sociocultural attitudes towards appearance

quiestionnaire-4. 0.84)

Some were considered

ethnicity, parenta

ethnicity but not

considered SES, family dynamics, relationship, etc.

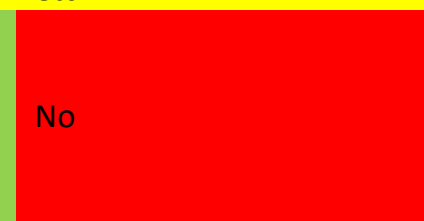

Yes (see \#4)

Descriptive statistics and correlation; independent samples t-test
Dysmorphic concern questionnaire (DCQ);

psychologic distress

(depression, anxiety, and stress scale, DASS-21); desire to obtain future beauty services ( 5 scale questionnaire)

No coefficient alpha noted

occupation, gender, duration of video call use, should have included SES, relationship status

Validity checks were conducted to ensure that groups completing the survey at each time point did not differ on variable of interest

See \#4

Welch's ANOVA, Bonferron corrected alpha, Pearson's correlation

Figure C3. Cross sectional studies (Joanna Briggs Institute, n.d.)

Were the criteria for inclusion in the sample clearly defined?
Pfund et al., 2020 
Were the study subjects and the setting described in detail?

Was the exposure measured in a valid and reliable way?

Were objective, standard criteria used for measurement of the condition?

Were confounding factors identified?

Were strategies to deal with confounding factors stated?

Were the outcomes measured in a valid and reliable way?
Setting was not described in detail, as it was administered for home use

Video chatting behaviours (Likert scale)

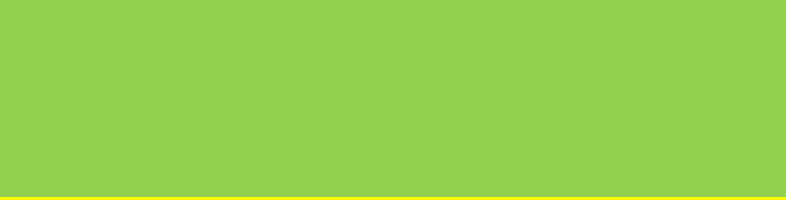

Self-objectification (8-item body surveillance subscale from the objectified body consciousness scale;

Cronbach's alpha 0.88); video chatting appearance comparison (physical appearance comparison scale; Cronbach's alpha 0.87); Appearance satisfaction (14item body parts satisfaction scale-revised; Cronbach's alpha 0.73)

Yes, attention check, reporting more than $24 \mathrm{~h} /$ day of video chatting,

Exclusion and statistical correction

See \#4 (not objective, but reliable and valid) 
Nurse Practitioner Open Journal

DOI 10.28984/npoj.vi2.361

Was appropriate statistical analysis

used?

R studio

Figure C4. Cross sectional studies (Joanna Briggs Institute, n.d.) 
Is there congruity between the stated philosophical perspective and the research methodology?

Is there congruity between the research methodology and the research question or objectives?

Is there congruity between the research methodology and the methods used to collect data?

Is there congruity between the research methodology and the representation and analysis of data?

Is there congruity between the research methodology and the interpretation of results?

Is there a statement locating the researcher culturally or theoretically?

Is the influence of the researcher on the research, and vice- versa, addressed?

Are participants, and their voices, adequately represented?

Is the research ethical according to current criteria or, for recent studies, and is there evidence of ethical approval by an appropriate body?
Tripartite model, mixed methods but no mention of qualitative research methodology

No mention of research methodology, so unable to assess, however appears to be phenomenological (understand the social media use, social comparison)

Yes. Qualitative study. Employed recorder and facilitator for a focus group

Yes - individual responses are shown

Yes - understanding the social media use and what it means for social comparison, body image

Yes - information about researcher ethnicity, age, background and experience is noted

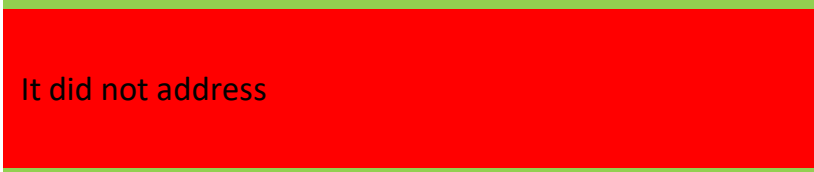

Yes, a table representing qualitative themes by group

Yes, approved by REB 
Do the conclusions drawn in the research report flow from the analysis, or interpretation, of the data?

Figure C5. Qualitative research (Joanna Briggs Institute, n.d.)

Was true randomization used for assignment of participants to treatment groups?

Was allocation to treatment groups concealed?

Were treatment groups similar at the baseline?

Were participants blind to treatment assignment?

Were those delivering treatment blind to treatment assignment?

Were outcomes assessors blind to treatment assignment?

Were treatment groups treated identically other than the intervention of interest?

\begin{tabular}{|c|c|}
\hline Fox et al., 2021 & Mills et al., 2018 \\
\hline $\begin{array}{l}\text { Unknown. Randomization process was } \\
\text { not described }\end{array}$ & $\begin{array}{l}\text { Unknown. Randomization process } \\
\text { not described }\end{array}$ \\
\hline Yes & Yes \\
\hline $\begin{array}{l}\text { Yes. People were recruited for course } \\
\text { credit and received same instructions }\end{array}$ & Yes \\
\hline $\begin{array}{l}\text { No. Unable to blind treatment } \\
\text { assignment due to the nature of the } \\
\text { study }\end{array}$ & $\begin{array}{l}\text { Unable to blind treatment } \\
\text { assignment } d / t \text { the nature of the } \\
\text { study, but all involved the use of } \\
\text { iPads which could reduce participant } \\
\text { bias }\end{array}$ \\
\hline No & No \\
\hline unknown & unknown \\
\hline $\begin{array}{l}\text { Yes. Everyone completed the same task } \\
\text { except for the intervention itself }\end{array}$ & $\begin{array}{l}\text { Yes, each went in a partition without } \\
\text { personal belongings and used } \\
\text { researcher provided iPad to } \\
\text { complete task }\end{array}$ \\
\hline
\end{tabular}

Yes, but would have been nice to have individual interviews with students as well
Kleemans et al., 2018

Unknown. Randomization process not described

Measured baseline characteristics are similar, but survey done independently, leaving room for variation

es, retouched vs. untouched image with all other factors equal

Unknown

\section{Unknown}

Likely, the intervention was completed individually so there can be differences not accounted for 
Was follow up complete and if not, were differences between groups in terms of their follow up adequately described and analyzed?

Were participants analyzed in the groups to which they were randomized?

Were outcomes measured in the same way for treatment groups?

Were outcomes measured in a reliable way?

Was appropriate statistical analysis used?
Yes, everyone was accounted for in the study

Yes, two-way MANCOVA (test the main and interaction effect), ANCOVA (main effect of each dependent variable independently), two-way ANVOCA (for social aggression)

Yes. Pre-task mood using (positive and negative affect schedule, PANAS), \# of pictures taken, state of self-

objectification (twenty statements test TST), post task mood (PANAS), selfesteem (20-item state self-esteem scale), social aggression via derogatory tags

Yes (see above)

Yes, see \#9

SPSS

Repeated measures MANOVA.

Significant multivariate 3-way

interactions are further analyzed

using repeated measures ANOVA

t-test

Yes, mood and body image (visual analogue scales)

Yes, body image (Body image state scale) and social comparison tendency (lowa-Netherland's comparison orientation measure)

VAS is a well validated scale but no coefficient stated for the assessment tool

Yes, see \#9
Methods have been used in previous studies. Cronbach alpha coefficient $>0.83$

Yes, see \#9 
Nurse Practitioner Open Journal

DOI 10.28984/npoj.vi2.361

Was the trial design

appropriate, and any deviations

from the standard RCT design

(individual randomization,

parallel groups) accounted for

in the conduct and analysis of

Figure C6. Randomized control trial (Joanna Briggs Institute, n.d.)
Yes, although randomization process
is unknown
Yes, but randomization process is
unknown

Unable to assess randomization process 